\title{
ACCURACY AND RELIABILITY OF A MODEL FOR A GAUSSIAN HOMOGENEOUS AND ISOTROPIC RANDOM FIELD \\ IN THE SPACE $L_{p}(\mathbb{T}), p \geq 1$
}

UDC 519.21

\author{
N. V. TROSHKI
}

\begin{abstract}
A model is constructed for a Gaussian homogeneous isotropic random field that approximates it with a given accuracy and reliability in the space $L_{p}(T)$, $p \geq 1$. The theory of the spaces $\operatorname{Sub}(\Omega)$ is used for studying such a model.
\end{abstract}

\section{INTRODUCTION}

There are many methods for modeling random fields (an interested reader may consult [1, 2] and [3, 4]). The methods for modeling random fields with a given accuracy and reliability in some function spaces, including Orlicz spaces $L_{U}(\mathbb{T})$ and $C(\mathbb{T})$, are considered in [5] and 6]-[11. Models of some stochastic processes are constructed in [12] and 13 for the spaces $D_{V, W}$ and in 14 for the space $F_{\psi}(\Omega)$.

This paper aims at constructing models for a homogeneous and isotropic Gaussian random field $X$ that approximate $X$ with a given reliability and accuracy in the space $L_{p}(\mathbb{T}), p \geq 1$. We construct models of a mean square continuous homogeneous isotropic Gaussian random field.

The paper consists of four main sections. Section 2 contains main definitions and known results that are used in the paper. Section 3 is devoted to constructing models of a homogeneous isotropic random field. In Section 4, we find new bounds for Bessel functions. The accuracy and reliability of models for random fields are discussed in Section 5 .

\section{MAin DEFinitions AND PROPERTIES}

2.1. Sub-Gaussian random variables and their properties. Let $\{\Omega, \mathbf{B}, \mathrm{P}\}$ be a standard probability space.

Definition 2.1 ([15]). A random variable $\xi$ is called sub-Gaussian if there exists s constant $a \geq 0$ such that

$$
E \exp \{\lambda \xi\} \leq \exp \left\{\frac{a^{2} \lambda^{2}}{2}\right\}
$$

for all $\lambda \in \mathbf{R}$.

The space of all sub-Gaussian random variables defined on a standard probability space $\{\Omega, \mathbf{B}, \mathrm{P}\}$ is denoted by $\operatorname{Sub}(\Omega)$. Note that $\operatorname{Sub}(\Omega)$ is a Banach space with respect

2010 Mathematics Subject Classification. Primary 60G15; Secondary 60G07.

Key words and phrases. Gaussian random fields, homogeneous and isotropic fields, models of random fields, accuracy and reliability. 
to the norm

$$
\tau(\xi)=\sup _{\lambda \neq 0}\left[(2 \ln \mathrm{E} \exp \{\lambda \xi\}) / \lambda^{2}\right]^{1 / 2} .
$$

Lemma 2.1 ([15]). Let $\xi_{1}, \xi_{2}, \ldots, \xi_{n}$ be independent sub-Gaussian random variables. Then

$$
\tau^{2}\left(\sum_{k=1}^{n} \xi_{k}\right) \leq \sum_{k=1}^{n} \tau^{2}\left(\xi_{k}\right)
$$

Lemma 2.2 ([15]). Let $\xi$ be a centered random variable such that $\mathrm{E} \xi^{2 k+1}=0$ and

$$
\theta(\xi)=\sup _{k \geq 1}\left[\frac{2^{k} k !}{(2 k) !} \mathrm{E} \xi^{2 k}\right]^{\frac{1}{2 k}}<\infty .
$$

Then $\xi \in \operatorname{Sub}(\Omega)$ and $\tau(\xi) \leq \theta(\xi)$.

\subsection{Homogeneous and isotropic random fields.}

Definition 2.2 ([16]). A random field $X=\left\{X(t), t \in \mathbf{R}^{2}\right\}$ is called wide sense homogeneous in the space $\mathbf{R}^{2}$ if $\mathrm{E} X(t)=$ const, $t \in \mathbf{R}^{2}$, and

$$
\mathrm{E} X(t) X(s)=B(t-s)=\int_{\mathbf{R}^{2}} e^{i(\lambda, t-s)} d F(\lambda), \quad t, s \in \mathbf{R}^{2} .
$$

Definition $2.3([16])$. Let $S O(2)$ be the group of rotations in $\mathbf{R}^{2}$. A homogeneous random field $X(t), t \in \mathbf{R}^{2}$, is called isotropic if

$$
\mathrm{E} X(t) X(s)=\mathrm{E} X(g t) X(g s)
$$

for any element $g$ of the group $S O(2)$ and for all $t, s \in \mathbf{R}^{2}$.

\section{Constructing a model of a homogeneous And isotropic RAndom Field}

Let $X=\{X(t, x), t \in \mathbf{R}, x \in[0,2 \pi]\}$ be a square mean continuous real valued homogeneous isotropic random field defined on $\mathbf{R}^{2}$. Then, similarly to the case of a complex valued field considered in [16], the following representation:

$$
X(t, x)=\sum_{k=1}^{\infty} \cos (k x) \int_{0}^{\infty} J_{k}(t \lambda) d \eta_{1, k}(\lambda)+\sum_{k=1}^{\infty} \sin (k x) \int_{0}^{\infty} J_{k}(t \lambda) d \eta_{2, k}(\lambda)
$$

holds where $\eta_{i, k}(\lambda), i=1,2, k=1,2, \ldots$, are independent Gaussian processes with independent increments, $\mathrm{E} \eta_{i, k}(\lambda)=0, \mathrm{E}\left(\eta_{i, k}(b)-\eta_{i, k}(c)\right)^{2}=F(b)-F(c), b>c, F(\lambda)$ is the spectral function of the field $X$, and $J_{k}(u)$ is the Bessel function. It is known that

$$
J_{k}(u)=\frac{1}{\pi} \int_{0}^{\pi} \cos (k \varphi-u \sin \varphi) d \varphi
$$

(see [17]).

Consider a certain partition $L=\left\{\lambda_{0}, \ldots, \lambda_{N}\right\}$ of the set $[0, \infty)$ such that $\lambda_{0}=0$, $\lambda_{l}<\lambda_{l+1}, \lambda_{N-1}=\Lambda, \lambda_{N}=\infty$ and $C=\max _{0<l \leq N-2} \frac{\lambda_{l+1}}{\lambda_{l}}<\infty$.

Then

$$
\hat{X}(t, x)=\sum_{k=1}^{M} \cos (k x) \sum_{l=0}^{N-1} \eta_{1, k, l} J_{k}\left(t \zeta_{l}\right)+\sum_{k=1}^{M} \sin (k x) \sum_{l=0}^{N-1} \eta_{2, k, l} J_{k}\left(t \zeta_{l}\right)
$$

is viewed as a model for the field $X(t, x)$, where

$$
\eta_{i, k, l}=\int_{\lambda_{l}}^{\lambda_{l+1}} d \eta_{i, k}(\lambda), \quad i=1,2
$$


are independent Gaussian random variables such that $\mathrm{E} \eta_{i, k, l}=0$,

$$
\mathrm{E} \eta_{i, k, l}^{2}=F\left(\lambda_{l+1}\right)-F\left(\lambda_{l}\right)=b_{l}^{2},
$$

$\zeta_{l}$ are independent random variables being independent of $\eta_{i, k, l}$ and that assume values in the intervals $\left[\lambda_{l}, \lambda_{l+1}\right]$, and where $b_{l}^{2}>0$ are such that

$$
F_{l}(\lambda)=\mathrm{P}\left\{\zeta_{l}<\lambda\right\}=\frac{F(\lambda)-F\left(\lambda_{l}\right)}{F\left(\lambda_{l+1}\right)-F\left(\lambda_{l}\right)} .
$$

If $b_{l}^{2}=0$, then $\zeta_{l}=0$ with probability one. For the sake of simplicity, we assume that $b_{l}^{2}>0, l=0,1, \ldots, N-1$.

Thus $\hat{X}(t, x)$ is given by

$$
\begin{aligned}
\hat{X}(t, x)= & \sum_{k=1}^{M} \cos (k x) \sum_{l=0}^{N-1} \int_{\lambda_{l}}^{\lambda_{l+1}} J_{k}\left(t \zeta_{l}\right) d \eta_{1, k}(\lambda) \\
& +\sum_{k=1}^{M} \sin (k x) \sum_{l=0}^{N-1} \int_{\lambda_{l}}^{\lambda_{l+1}} J_{k}\left(t \zeta_{l}\right) d \eta_{2, k}(\lambda) .
\end{aligned}
$$

It is clear that $X(t, x)$ can be rewritten as follows:

$$
\begin{aligned}
X(t, x)= & \sum_{k=1}^{\infty} \cos (k x) \sum_{l=0}^{N-1} \int_{\lambda_{l}}^{\lambda_{l+1}} J_{k}(t \lambda) d \eta_{1, k}(\lambda) \\
& +\sum_{k=1}^{\infty} \sin (k x) \sum_{l=0}^{N-1} \int_{\lambda_{l}}^{\lambda_{l+1}} J_{k}(t \lambda) d \eta_{2, k}(\lambda) .
\end{aligned}
$$

\section{Preliminary Results}

Put

(3)

$$
\begin{aligned}
\chi_{M}(t, x)= & X(t, x)-\hat{X}(t, x) \\
= & \sum_{k=1}^{M} \cos (k x) \sum_{l=0}^{N-1} \int_{\lambda_{l}}^{\lambda_{l+1}}\left(J_{k}(t \lambda)-J_{k}\left(t \zeta_{l}\right)\right) d \eta_{1, k}(\lambda) \\
& +\sum_{k=M+1}^{\infty} \cos (k x) \int_{0}^{\infty} J_{k}(t \lambda) d \eta_{1, k}(\lambda) \\
& +\sum_{k=1}^{M} \sin (k x) \sum_{l=0}^{N-1} \int_{\lambda_{l}}^{\lambda_{l+1}}\left(J_{k}(t \lambda)-J_{k}\left(t \zeta_{l}\right)\right) d \eta_{2, k}(\lambda) \\
& +\sum_{k=M+1}^{\infty} \sin (k x) \int_{0}^{\infty} J_{k}(t \lambda) d \eta_{2, k}(\lambda) \\
= & : \chi_{M, 1}(t, x)+\chi_{M, 2}(t, x) .
\end{aligned}
$$

Then

$$
\tau\left(\chi_{M}(t, x)\right) \leq \tau\left(\chi_{M, 1}(t, x)\right)+\tau\left(\chi_{M, 2}(t, x)\right)
$$


and

$$
\begin{aligned}
\tau^{2}\left(\chi_{M, 1}(t, x)\right) \leq & \tau^{2}\left(\sum_{k=1}^{M} \cos (k x) \sum_{l=0}^{N-1} \int_{\lambda_{l}}^{\lambda_{l+1}}\left(J_{k}(t \lambda)-J_{k}\left(t \zeta_{l}\right)\right) d \eta_{1, k}(\lambda)\right) \\
& +\tau^{2}\left(\sum_{k=M+1}^{\infty} \cos (k x) \int_{0}^{\infty} J_{k}(t \lambda) d \eta_{1, k}(\lambda)\right), \\
\tau^{2}\left(\chi_{M, 2}(t, x)\right) \leq & \tau^{2}\left(\sum_{k=1}^{M} \sin (k x) \sum_{l=0}^{N-1} \int_{\lambda_{l}}^{\lambda_{l+1}}\left(J_{k}(t \lambda)-J_{k}\left(t \zeta_{l}\right)\right) d \eta_{2, k}(\lambda)\right) \\
& +\tau^{2}\left(\sum_{k=M+1}^{\infty} \sin (k x) \int_{0}^{\infty} J_{k}(t \lambda) d \eta_{2, k}(\lambda)\right) .
\end{aligned}
$$

Lemma 4.1. For all $\frac{1}{2}<\alpha \leq 1$,

$$
\left|J_{k}(u)\right| \leq 2^{1-\alpha}|u|^{\alpha} \pi^{\alpha} \frac{1}{k^{\alpha}}
$$

Proof. We have

$$
\begin{aligned}
\left|J_{k}(u)\right| & =\frac{1}{\pi}\left|\int_{0}^{\pi} \cos (k \varphi-u \sin \varphi) d \varphi\right| \\
& =\frac{1}{\pi}\left|\int_{0}^{\pi} \cos (k \varphi) \cos (u \sin \varphi) d \varphi+\int_{0}^{\pi} \sin (k \varphi) \sin (u \sin \varphi) d \varphi\right| \\
& =\frac{1}{\pi}\left|I_{1}+I_{2}\right| \\
& \leq \frac{1}{\pi}\left(\left|I_{1}\right|+\left|I_{2}\right|\right)
\end{aligned}
$$

Since the integrand in the integral $I_{1}$ is an even and periodic function with period $2 \pi$, we can transform $I_{1}$ as follows:

$$
\begin{aligned}
I_{1} & =\int_{0}^{\pi} \cos (k \varphi) \cos (u \sin \varphi) d \varphi \\
& =\frac{1}{2} \int_{-\pi}^{\pi} \cos (k \varphi) \cos (u \sin \varphi) d \varphi \\
& =\frac{1}{2} \int_{-\pi}^{\pi} \cos \left(k\left(\varphi+\frac{\pi}{k}\right)\right) \cos \left(u \sin \left(\varphi+\frac{\pi}{k}\right)\right) d \varphi \\
& =\frac{1}{2} \int_{-\pi}^{\pi} \cos (k \varphi+\pi) \cos \left(u \sin \left(\varphi+\frac{\pi}{k}\right)\right) d \varphi \\
& =-\frac{1}{2} \int_{-\pi}^{\pi} \cos (k \varphi) \cos \left(u \sin \left(\varphi+\frac{\pi}{k}\right)\right) d \varphi .
\end{aligned}
$$

Then the integral $I_{1}$ is written as follows:

$$
\begin{aligned}
I_{1}= & -\frac{1}{4} \int_{-\pi}^{\pi} \cos (k \varphi) \cos \left(u \sin \left(\varphi+\frac{\pi}{k}\right)\right) d \varphi \\
& +\frac{1}{4} \int_{-\pi}^{\pi} \cos (k \varphi) \cos (u \sin \varphi) d \varphi
\end{aligned}
$$


We are going to obtain a bound for $\left|I_{1}\right|$. Indeed,

$$
\begin{aligned}
& \left|I_{1}\right|=\left|-\frac{1}{4} \int_{-\pi}^{\pi} \cos (k \varphi) \cos \left(u \sin \left(\varphi+\frac{\pi}{k}\right)\right) d \varphi+\frac{1}{4} \int_{-\pi}^{\pi} \cos (k \varphi) \cos (u \sin \varphi) d \varphi\right| \\
& \leq \frac{1}{4} \int_{-\pi}^{\pi}\left|\cos (k \varphi)\left(\cos (u \sin \varphi)-\cos \left(u \sin \left(\varphi+\frac{\pi}{k}\right)\right)\right)\right| d \varphi \\
& =\frac{1}{4} \int_{-\pi}^{\pi} \mid \cos (k \varphi) \cdot 2 \sin \left(\frac{u\left(\sin \left(\varphi+\frac{\pi}{k}\right)-\sin \varphi\right)}{2}\right) \\
& \times \sin \left(\frac{u\left(\sin \left(\varphi+\frac{\pi}{k}\right)+\sin \varphi\right)}{2}\right) \mid d \varphi \\
& \leq \frac{1}{2} \int_{-\pi}^{\pi}\left|\sin \left(\frac{u\left(\sin \left(\varphi+\frac{\pi}{k}\right)-\sin \varphi\right)}{2}\right)\right| d \varphi \\
& \leq \frac{1}{2} \int_{-\pi}^{\pi}\left|\frac{u}{2}\right|^{\alpha}\left|\sin \left(\varphi+\frac{\pi}{k}\right)-\sin \varphi\right|^{\alpha} d \varphi \\
& =\frac{1}{2} \int_{-\pi}^{\pi}\left|\frac{u}{2}\right|^{\alpha}\left|2 \cos \left(\frac{2 \varphi+\frac{\pi}{k}}{2}\right) \cdot \sin \frac{\pi}{2 k}\right|^{\alpha} d \varphi \\
& \leq \frac{1}{2} \int_{-\pi}^{\pi}|u|^{\alpha}\left|\sin \frac{\pi}{2 k}\right|^{\alpha} d \varphi \\
& =|u|^{\alpha} \cdot \pi\left|\sin \frac{\pi}{2 k}\right|^{\alpha} \\
& \leq \pi \cdot|u|^{\alpha}\left(\frac{\pi}{2 k}\right)^{\alpha} \text {. }
\end{aligned}
$$

For the integral $I_{2}$,

$$
\begin{aligned}
I_{2} & =\int_{0}^{\pi} \sin (k \varphi) \sin (u \sin \varphi) d \varphi \\
& =\frac{1}{2} \int_{-\pi}^{\pi} \sin (k \varphi) \sin (u \sin \varphi) d \varphi \\
& =\frac{1}{2} \int_{-\pi}^{\pi} \sin \left(k\left(\varphi+\frac{\pi}{k}\right)\right) \sin \left(u \sin \left(\varphi+\frac{\pi}{k}\right)\right) d \varphi \\
& \left.=\frac{1}{2} \int_{-\pi}^{\pi} \sin (k \varphi-\pi)\right) \sin \left(u \sin \left(\varphi+\frac{\pi}{k}\right)\right) d \varphi \\
& =-\frac{1}{2} \int_{-\pi}^{\pi} \sin (k \varphi) \sin \left(u \sin \left(\varphi+\frac{\pi}{k}\right)\right) d \varphi
\end{aligned}
$$

As in the case of $I_{1}$, the integral $I_{2}$ is transformed to the following form:

$$
\begin{aligned}
I_{2}= & -\frac{1}{4} \int_{-\pi}^{\pi} \sin (k \varphi) \sin \left(u \sin \left(\varphi+\frac{\pi}{k}\right)\right) d \varphi \\
& +\frac{1}{4} \int_{-\pi}^{\pi} \sin (k \varphi) \sin (u \sin \varphi) d \varphi .
\end{aligned}
$$

Then $\left|I_{2}\right|$ admits the following bound:

$$
\left|I_{2}\right| \leq \pi \cdot|u|^{\alpha}\left(\frac{\pi}{2 k}\right)^{\alpha}
$$


whence

$$
\begin{aligned}
\left|J_{k}(u)\right| & =\frac{1}{\pi}\left|I_{1}+I_{2}\right| \\
& \leq \frac{1}{\pi}\left(\pi \cdot|u|^{\alpha}\left(\frac{\pi}{2 k}\right)^{\alpha}+\pi \cdot|u|^{\alpha}\left(\frac{\pi}{2 k}\right)^{\alpha}\right) \\
& =2^{1-\alpha} \cdot|u|^{\alpha} \pi^{\alpha} \cdot \frac{1}{k^{\alpha}},
\end{aligned}
$$

where $\frac{1}{2}<\alpha \leq 1$.

Lemma 4.2. For all $\frac{1}{2}<\alpha \leq 1$,

$$
\left|J_{k}(t \lambda)-J_{k}(t u)\right| \leq 4^{1-\alpha} t^{\alpha}|\lambda-u|^{\alpha} \pi^{\alpha} \cdot \frac{1}{k^{\alpha}}\left(1+\frac{t^{\alpha}|\lambda+u|^{\alpha}}{2^{\alpha}}\right) .
$$

Proof. Using the integrals $I_{1}$ and $I_{2}$ (evaluated in the proof of Lemma 4.1), we get

$$
\begin{aligned}
\left|J_{k}(t \lambda)-J_{k}(t u)\right|=\frac{1}{\pi} \mid( & -\frac{1}{4} \int_{-\pi}^{\pi} \cos (k \varphi) \cos \left(t \lambda \sin \left(\varphi+\frac{\pi}{k}\right)\right) d \varphi \\
& +\frac{1}{4} \int_{-\pi}^{\pi} \cos (k \varphi) \cos (t \lambda \sin \varphi) d \varphi \\
& +\frac{1}{4} \int_{-\pi}^{\pi} \cos (k \varphi) \cos \left(t u \sin \left(\varphi+\frac{\pi}{k}\right)\right) d \varphi \\
+ & -\frac{1}{4} \int_{-\pi}^{\pi} \sin (k \varphi) \sin \left(t \lambda \sin \left(\varphi+\frac{\pi}{k}\right)\right) d \varphi \\
& +\frac{1}{4} \int_{-\pi}^{\pi} \sin (k \varphi) \sin (t \lambda \sin \varphi) d \varphi \\
& +\frac{1}{4} \int_{-\pi}^{\pi} \sin (k \varphi) \sin \left(t u \sin \left(\varphi+\frac{1}{k}\right)\right) d \varphi \\
=\frac{1}{\pi} \mid S_{1} & +S_{2} \mid \begin{array}{l}
\frac{1}{4}\left(\left|S_{1}\right|\right. \\
\leq
\end{array} \\
\left.\leq S_{2} \mid\right) . & \sin (k \varphi) \sin (t u \sin \varphi) d \varphi) \mid
\end{aligned}
$$

Then we find a bound for $\left|S_{1}\right|$ :

$$
\begin{aligned}
\left|S_{1}\right|=\mid & -\frac{1}{4} \int_{-\pi}^{\pi} \cos (k \varphi) \cos \left(t \lambda \sin \left(\varphi+\frac{\pi}{k}\right)\right) d \varphi \\
& +\frac{1}{4} \int_{-\pi}^{\pi} \cos (k \varphi) \cos (t \lambda \sin \varphi) d \varphi+\frac{1}{4} \int_{-\pi}^{\pi} \cos (k \varphi) \cos \left(t u \sin \left(\varphi+\frac{\pi}{k}\right)\right) d \varphi \\
\leq \frac{1}{4} \int_{-\pi}^{\pi}|\cos (k \varphi)| \mid \cos (t \lambda \sin \varphi)-\cos (t u \sin \varphi) & -\frac{1}{4} \int_{-\pi}^{\pi} \cos (k \varphi) \cos (t u \sin \varphi) d \varphi \mid \\
& \quad-\left(\cos \left(t \lambda \sin \left(\varphi+\frac{\pi}{k}\right)\right)-\cos \left(t u \sin \left(\varphi+\frac{\pi}{k}\right)\right)\right) \mid d \varphi
\end{aligned}
$$




$$
\begin{aligned}
& =\frac{1}{2} \int_{-\pi}^{\pi}|\cos (k \varphi)| \mid \sin \frac{t(u+\lambda) \sin \varphi}{2} \sin \frac{t(u-\lambda) \sin \varphi}{2} \\
& -\sin \frac{t(u+\lambda) \sin \left(\varphi+\frac{\pi}{k}\right)}{2} \sin \frac{t(u-\lambda) \sin \left(\varphi+\frac{\pi}{k}\right)}{2} \mid d \varphi \\
& =\frac{1}{2} \int_{-\pi}^{\pi}|\cos (k \varphi)| \sin \frac{t(u+\lambda) \sin \varphi}{2}\left(\sin \frac{t(u-\lambda) \sin \varphi}{2}-\sin \frac{t(u-\lambda) \sin \left(\varphi+\frac{\pi}{k}\right)}{2}\right) \\
& +\sin \frac{t(u-\lambda) \sin \left(\varphi+\frac{\pi}{k}\right)}{2} \\
& \times\left(\sin \frac{t(u+\lambda) \sin \varphi}{2}-\sin \frac{t(u+\lambda) \sin \left(\varphi+\frac{\pi}{k}\right)}{2}\right) \mid d \varphi \\
& =\int_{-\pi}^{\pi}|\cos (k \varphi)| \mid \sin \frac{t(u+\lambda) \sin \varphi}{2} \cdot \cos \frac{t(u-\lambda)\left(\sin \varphi+\sin \left(\varphi+\frac{\pi}{k}\right)\right)}{4} \\
& \times \sin \frac{t(u-\lambda)\left(\sin \varphi-\sin \left(\varphi+\frac{\pi}{k}\right)\right)}{4} \\
& +\sin \frac{t(u-\lambda) \sin \left(\varphi+\frac{w \pi}{k}\right)}{2} \cdot \cos \frac{t(u+\lambda)\left(\sin \varphi+\sin \left(\varphi+\frac{\pi}{k}\right)\right)}{4} \\
& \times \sin \frac{t(u+\lambda)\left(\sin \varphi-\sin \left(\varphi+\frac{\pi}{k}\right)\right)}{4} \mid d \varphi \\
& \leq \int_{-\pi}^{\pi}\left(\left|\sin \frac{t(u-\lambda)\left(\sin \varphi-\sin \left(\varphi+\frac{\pi}{k}\right)\right)}{4}\right|\right. \\
& \left.+\left|\sin \frac{t(u-\lambda) \sin \left(\varphi+\frac{\pi}{k}\right)}{2} \sin \frac{t(u+\lambda)\left(\sin \varphi-\sin \left(\varphi+\frac{\pi}{k}\right)\right)}{4}\right|\right) d \varphi \\
& \leq 2 \pi\left(\frac{t^{\alpha}|\lambda-u|^{\alpha}\left(\frac{\pi}{2 k}\right)^{\alpha}}{2^{\alpha}}+\frac{t^{\alpha}|\lambda-u|^{\alpha} t^{\alpha}|\lambda+u|^{\alpha}\left(\frac{\pi}{2 k}\right)^{\alpha}}{2^{\alpha} \cdot 2^{\alpha}}\right) \\
& =2 \pi\left(\frac{t}{2}\right)^{\alpha}|\lambda-u|^{\alpha}\left(\frac{\pi}{2 k}\right)^{\alpha}\left(1+\frac{t^{\alpha}|\lambda+u|^{\alpha}}{2^{\alpha}}\right) .
\end{aligned}
$$

Similarly, we obtain a bound for $\left|S_{2}\right|$ :

$$
\begin{aligned}
\left|S_{2}\right|=\mid- & \frac{1}{4} \int_{-\pi}^{\pi} \sin (k \varphi) \sin \left(t \lambda \sin \left(\varphi+\frac{\pi}{k}\right)\right) d \varphi \\
& +\frac{1}{4} \int_{-\pi}^{\pi} \sin (k \varphi) \sin (t \lambda \sin \varphi) d \varphi \\
& +\frac{1}{4} \int_{-\pi}^{\pi} \sin (k \varphi) \sin \left(t u \sin \left(\varphi+\frac{\pi}{k}\right)\right) d \varphi-\frac{1}{4} \int_{-\pi}^{\pi} \sin (k \varphi) \sin (t u \sin \varphi) d \varphi \mid \\
\leq & \frac{1}{4} \int_{-\pi}^{\pi}|\sin (k \varphi)| \mid(\sin (t \lambda \sin \varphi)-\sin (t u \sin \varphi)) \\
= & \quad-\left(\sin \left(t \lambda \sin \left(\varphi+\frac{\pi}{k}\right)\right)-\sin \left(t u \sin \left(\varphi+\frac{\pi}{k}\right)\right)\right) \mid d \varphi \\
& \quad-\cos \frac{t(\lambda+u) \sin \left(\varphi+\frac{\pi}{k}\right)}{2} \sin \frac{t(\lambda-u) \sin \left(\varphi+\frac{\pi}{k}\right)}{2} \mid d \varphi
\end{aligned}
$$




$$
\begin{aligned}
& =\frac{1}{2} \int_{-\pi}^{\pi}|\sin (k \varphi)| \mid \cos \frac{t(\lambda+u) \sin \varphi}{2}\left(\sin \frac{t(\lambda-u) \sin \varphi}{2}-\sin \frac{t(\lambda-u) \sin \left(\varphi+\frac{\pi}{k}\right)}{2}\right) \\
& +\sin \frac{t(\lambda-u) \sin \left(\varphi+\frac{\pi}{k}\right)}{2} \\
& \times\left(\cos \frac{t(\lambda+u) \sin \varphi}{2}-\cos \frac{t(\lambda+u) \sin \left(\varphi+\frac{\pi}{k}\right)}{2}\right) \mid d \varphi \\
& \leq \int_{-\pi}^{\pi}|\sin (k \varphi)|\left(\mid \cos \frac{t(\lambda+u) \sin \varphi}{2} \cos \frac{t(\lambda-u)\left(\sin \varphi+\sin \left(\varphi+\frac{\pi}{k}\right)\right)}{4}\right. \\
& \times \sin \frac{t(\lambda-u)\left(\sin \varphi-\sin \left(\varphi+\frac{\pi}{k}\right)\right)}{4} \mid \\
& +\mid \sin \frac{t(\lambda-u) \sin \left(\varphi+\frac{\pi}{k}\right)}{2} \sin \frac{t(\lambda+u)\left(\sin \varphi+\sin \left(\varphi+\frac{\pi}{k}\right)\right)}{4} \\
& \left.\times \sin \frac{t(\lambda+u)\left(\sin \left(\varphi+\frac{\pi}{k}\right)-\sin \varphi\right)}{4} \mid\right) d \varphi \\
& \leq \int_{-\pi}^{\pi}\left(\left|\sin \frac{t(\lambda-u)\left(\sin \varphi-\sin \left(\varphi+\frac{\pi}{k}\right)\right)}{4}\right|\right. \\
& \left.+\left|\sin \frac{t(\lambda-u) \sin \left(\varphi+\frac{\pi}{k}\right)}{2} \sin \frac{t(\lambda+u)\left(\sin \left(\varphi+\frac{\pi}{k}\right)-\sin \varphi\right)}{4}\right|\right) d \varphi \\
& \leq 2 \pi\left(\frac{t^{\alpha}|\lambda-u|^{\alpha}\left(\frac{\pi}{2 k}\right)^{\alpha}}{2^{\alpha}}+\frac{t^{\alpha}|\lambda-u|^{\alpha} t^{\alpha}|\lambda+u|^{\alpha}\left(\frac{\pi}{2 k}\right)^{\alpha}}{2^{\alpha} \cdot 2^{\alpha}}\right) \\
& =2 \pi\left(\frac{t}{2}\right)^{\alpha}|\lambda-u|^{\alpha}\left(\frac{\pi}{2 k}\right)^{\alpha}\left(1+\frac{t^{\alpha}|\lambda+u|^{\alpha}}{2^{\alpha}}\right) .
\end{aligned}
$$

Then

$$
\begin{aligned}
\left|J_{k}(t \lambda)-J_{k}(t u)\right| \leq & 2\left(\frac{t}{2}\right)^{\alpha}|\lambda-u|^{\alpha}\left(\frac{\pi}{2 k}\right)^{\alpha}\left(1+\frac{t^{\alpha}|\lambda+u|^{\alpha}}{2^{\alpha}}\right) \\
& +2\left(\frac{t}{2}\right)^{\alpha}|\lambda-u|^{\alpha}\left(\frac{\pi}{2 k}\right)^{\alpha}\left(1+\frac{t^{\alpha}|\lambda+u|^{\alpha}}{2^{\alpha}}\right) \\
= & 4^{1-\alpha} t^{\alpha}|\lambda-u|^{\alpha} \pi^{\alpha} \cdot \frac{1}{k^{\alpha}}\left(1+\frac{t^{\alpha}|\lambda+u|^{\alpha}}{2^{\alpha}}\right) .
\end{aligned}
$$

Lemma 4.3. For all $\frac{1}{2}<\alpha \leq 1$,

$$
\begin{aligned}
\tau^{2}\left(\sum_{k=1}^{M} \cos (k x) \sum_{l=0}^{N-1} \int_{\lambda_{l}}^{\lambda_{l+1}}\left(J_{k}(t \lambda)-J_{k}\left(t \zeta_{l}\right)\right) d \eta_{1, k}(\lambda)\right) \\
\leq \frac{1}{2 \alpha-1}\left(2 \alpha-\frac{1}{M^{2 \alpha-1}}\right) 2 \cdot 4^{2(1-\alpha)} \pi^{2 \alpha} t^{2 \alpha} \\
\quad \times \sum_{l=0}^{N-2}\left|\lambda_{l+1}-\lambda_{l}\right|^{2 \alpha}\left(b_{l}^{2}+\left(\frac{t(1+C)}{2}\right)^{2 \alpha} \int_{\lambda_{l}}^{\lambda_{l+1}} \lambda^{2 \alpha} d F(\lambda)\right) \\
+4 M(F(+\infty)-F(\Lambda)),
\end{aligned}
$$




$$
\begin{aligned}
\tau^{2}\left(\sum_{k=1}^{M} \sin (k x) \sum_{l=0}^{N-1} \int_{\lambda_{l}}^{\lambda_{l+1}}\left(J_{k}(t \lambda)-J_{k}\left(t \zeta_{l}\right)\right) d \eta_{2, k}(\lambda)\right) \\
\leq \frac{1}{2 \alpha-1}\left(2 \alpha-\frac{1}{M^{2 \alpha-1}}\right) 2 \cdot 4^{2(1-\alpha)} \pi^{2 \alpha} t^{2 \alpha} \\
\quad \times \sum_{l=0}^{N-2}\left|\lambda_{l+1}-\lambda_{l}\right|^{2 \alpha}\left(b_{l}^{2}+\left(\frac{t(1+C)}{2}\right)^{2 \alpha} \int_{\lambda_{l}}^{\lambda_{l+1}} \lambda^{2 \alpha} d F(\lambda)\right) \\
+4 M(F(+\infty)-F(\Lambda)),
\end{aligned}
$$

where $C=\max _{0<l \leq N-2} \frac{\lambda_{l+1}}{\lambda_{l}}$.

Proof. We have

$$
\begin{aligned}
& \tau^{2}\left(\sum_{k=1}^{M} \cos (k x) \sum_{l=0}^{N-1} \int_{\lambda_{l}}^{\lambda_{l+1}}\left(J_{k}(t \lambda)-J_{k}\left(t \zeta_{l}\right)\right) d \eta_{1, k}(\lambda)\right) \\
& \quad \leq \sum_{k=1}^{M} \sum_{l=0}^{N-1} \tau^{2}\left(\int_{\lambda_{l}}^{\lambda_{l+1}}\left(J_{k}(t \lambda)-J_{k}\left(t \zeta_{l}\right)\right) d \eta_{1, k}(\lambda)\right) \\
& \quad \leq \sum_{k=1}^{M} \sum_{l=0}^{N-1} \theta^{2}\left(\int_{\lambda_{l}}^{\lambda_{l+1}}\left(J_{k}(t \lambda)-J_{k}\left(t \zeta_{l}\right)\right) d \eta_{1, k}(\lambda)\right) \\
& \quad=\sum_{k=1}^{M} \sum_{l=0}^{N-1} \sup _{m \geq 1}\left[\frac{2^{m} \cdot m !}{(2 m) !} E\left(\int_{\lambda_{l}}^{\lambda_{l+1}}\left(J_{k}(t \lambda)-J_{k}\left(t \zeta_{l}\right)\right) d \eta_{1, k}(\lambda)\right)^{2 m}\right]^{\frac{1}{m}} .
\end{aligned}
$$

Since $\mathrm{E} \xi=0, \mathrm{E} \xi^{2 k+1}=0$, and

$$
\mathrm{E} \xi^{2 k}=\frac{(2 k) !}{2^{k} \cdot k !} \sigma^{2 k}
$$

for any centered Gaussian random variable $\xi$ and since the random variables $\zeta_{l}$ do not depend on $\eta_{i, k}(\lambda), i=1,2$, we obtain from Fubini's theorem and Lemma 4.2 that

$$
\begin{aligned}
\mathrm{E}\left(\int_{\lambda_{l}}^{\lambda_{l+1}}\left(J_{k}(t \lambda)-J_{k}\left(t \zeta_{l}\right)\right) d \eta_{1, k}(\lambda)\right)^{2 m} \\
\quad=\mathrm{E}_{\zeta_{l}}\left(\int_{\lambda_{l}}^{\lambda_{l+1}}\left(J_{k}(t \lambda)-J_{k}\left(t \zeta_{l}\right)\right) d \eta_{1, k}(\lambda)\right)^{2 m} \\
\quad \leq \frac{(2 m) !}{2^{m} \cdot m !} \mathrm{E}\left(\int_{\lambda_{l}}^{\lambda_{l+1}}\left|J_{k}(t \lambda)-J_{k}\left(t \zeta_{l}\right)\right|^{2} d F(\lambda)\right)^{m} \\
\leq \frac{(2 m) !}{2^{m} \cdot m !} \mathrm{E}\left(\int_{\lambda_{l}}^{\lambda_{l+1}}\left(4^{1-\alpha} t^{\alpha}\left|\lambda-\zeta_{l}\right|^{\alpha} \pi^{\alpha} \cdot \frac{1}{k^{\alpha}}\left(1+\frac{t^{\alpha}\left|\lambda+\zeta_{l}\right|^{\alpha}}{2^{\alpha}}\right)\right)^{2} d F(\lambda)\right)^{m} \\
=\frac{(2 m) !}{2^{m} \cdot m !} 4^{2 m(1-\alpha)} t^{2 m \alpha} \pi^{2 m \alpha}\left(\frac{1}{k}\right)^{2 m \alpha} \\
\quad \times \mathrm{E}\left(\int_{\lambda_{l}}^{\lambda_{l+1}}\left|\lambda-\zeta_{l}\right|^{2 \alpha}\left(1+\frac{t^{\alpha}\left|\lambda+\zeta_{l}\right|^{\alpha}}{2^{\alpha}}\right)^{2} d F(\lambda)\right)^{m}
\end{aligned}
$$




$$
\begin{aligned}
= & \frac{(2 m) !}{2^{m} \cdot m !} \cdot 4^{2 m(1-\alpha)} t^{2 m \alpha} \pi^{2 m \alpha}\left(\frac{1}{k}\right)^{2 m \alpha} \\
& \times \int_{\lambda_{l}}^{\lambda_{l+1}}\left(\int_{\lambda_{l}}^{\lambda_{l+1}}|\lambda-u|^{2 \alpha}\left(1+\frac{t^{\alpha}|\lambda+u|^{\alpha}}{2^{\alpha}}\right)^{2} d F(\lambda)\right)^{m} d F_{l}(u) \\
\leq & \frac{(2 m) !}{2^{m} \cdot m !} \cdot 4^{2 m(1-\alpha)} t^{2 m \alpha} \pi^{2 m \alpha}\left(\frac{1}{k}\right)^{2 m \alpha}\left|\lambda_{l+1}-\lambda_{l}\right|^{2 m \alpha} \\
& \times \int_{\lambda_{l}}^{\lambda_{l+1}}\left(\int_{\lambda_{l}}^{\lambda_{l+1}}\left(1+\frac{t^{\alpha} \lambda^{\alpha}\left|1+\frac{u}{\lambda}\right|^{\alpha}}{2^{\alpha}}\right)^{2} d F(\lambda)\right)^{m} d F_{l}(u) \\
\leq & \frac{(2 m) !}{2^{m} \cdot m !} \cdot 4^{2 m(1-\alpha)} t^{2 m \alpha} \pi^{2 m \alpha}\left(\frac{1}{k}\right)^{2 m \alpha}\left|\lambda_{l+1}-\lambda_{l}\right|^{2 m \alpha} \\
& \times\left(\int_{\lambda_{l}}^{\lambda_{l+1}}\left(1+\frac{t^{\alpha} \lambda^{\alpha}\left|1+\frac{\lambda_{l+1}}{\lambda_{l}}\right|^{\alpha}}{2^{\alpha}} d F(\lambda)\right)^{2}\right. \\
\leq & \frac{(2 m) !}{2^{m} \cdot m !} \cdot 4^{2 m(1-\alpha)} t^{2 m \alpha} \pi^{2 m \alpha}\left(\frac{1}{k}\right)^{2 m \alpha}\left|\lambda_{l+1}-\lambda_{l}\right|^{2 m \alpha} \\
& \times\left(\int_{\lambda_{l}}^{\lambda_{l+1}}\left(2+2 \cdot \frac{t^{2 \alpha} \lambda^{2 \alpha}(1+C)^{2 \alpha}}{2^{2 \alpha}}\right) d F(\lambda)\right)^{m} \\
= & \frac{(2 m) !}{2^{m} \cdot m !} \cdot 4^{2 m(1-\alpha)} t^{2 m \alpha} \pi^{2 m \alpha}\left(\frac{1}{k}\right)^{2 m \alpha}\left|\lambda_{l+1}-\lambda_{l}\right|^{2 m \alpha} \\
& \times\left(2 b_{l}^{2}+2 \cdot\left(\frac{t(1+C)}{2}\right)^{2 \alpha} \int_{\lambda_{l}}^{\lambda_{l+1}} \lambda^{2 \alpha} d F(\lambda)\right)^{m} \cdot
\end{aligned}
$$

Here the symbol $\mathrm{E}_{\zeta_{l}}$ denotes the conditional expectation with respect to $\zeta_{l}$. Note that

$$
\begin{aligned}
\left(\int_{\Lambda}^{\infty}\right. & \left.\left(\int_{\Lambda}^{\infty}\left|J_{k}(t \lambda)-J_{k}(t u)\right|^{2} d F(\lambda)\right)^{m} d F_{l}(u)\right)^{\frac{1}{m}} \\
= & \left(\int _ { \Lambda } ^ { \infty } \left(\int_{\Lambda}^{\infty} \mid \frac{1}{\pi}\left(\int_{0}^{\pi} \cos (k \varphi-t \lambda \sin \varphi) d \varphi\right.\right.\right. \\
& \left.\left.\left.-\int_{0}^{\pi} \cos (k \varphi-t u \sin \varphi) d \varphi\right)\left.\right|^{2} d F(\lambda)\right)^{m} d F_{l}(u)\right)^{\frac{1}{m}} \\
\leq & \left(\int _ { \Lambda } ^ { \infty } \left(\int _ { \Lambda } ^ { \infty } \left(\frac{1}{\pi} \int_{0}^{\pi} \mid \cos (k \varphi-t \lambda \sin \varphi)\right.\right.\right. \\
= & \left(\int_{\Lambda}^{\infty}\left(\int_{\Lambda}^{\infty}\left(\frac{1}{\pi} \int_{0}^{\pi} \mid 2 \sin \left(k \varphi-\frac{t(\lambda+u) \sin \varphi}{2}\right)^{2} d F(\lambda)\right)^{m} d F_{l}(u)\right)^{\frac{1}{m}}\right. \\
& \left.\left.\left.\times \sin \left(\frac{t(\lambda-u) \sin \varphi}{2}\right) \mid d \varphi\right)^{2} d F(\lambda)\right)^{m} d F_{l}(u)\right)^{\frac{1}{m}} .
\end{aligned}
$$


Therefore

$$
\begin{aligned}
& \left(\int_{\Lambda}^{\infty}\left(\int_{\Lambda}^{\infty}\left|J_{k}(t \lambda)-J_{k}(t u)\right|^{2} d F(\lambda)\right)^{m} d F_{l}(u)\right)^{\frac{1}{m}} \\
& \quad \leq 4\left(\int_{\Lambda}^{\infty}\left(\int_{\Lambda}^{\infty} d F(\lambda)\right)^{m} d F_{l}(u)\right)^{\frac{1}{m}}=4(F(+\infty)-F(\Lambda)),
\end{aligned}
$$

whence

$$
\begin{aligned}
& \tau^{2}\left(\sum_{k=1}^{M} \cos (k x) \sum_{l=0}^{N-1} \int_{\lambda_{l}}^{\lambda_{l+1}}\left(J_{k}(t \lambda)-J_{k}\left(t \zeta_{l}\right)\right) d \eta_{1, k}(\lambda)\right) \\
& \leq 4^{2(1-\alpha)} t^{2 \alpha} \pi^{2 \alpha} \\
& \quad \times \sum_{k=1}^{M} \sum_{l=0}^{N-1} \frac{1}{k^{2 \alpha}} \\
& \quad \times \sup _{m \geq 1}\left(\int_{\lambda_{l}}^{\lambda_{l+1}}\left(\int_{\lambda_{l}}^{\lambda_{l+1}}|\lambda-u|^{2 \alpha}\left(1+\frac{t^{\alpha}|\lambda+u|^{\alpha}}{2^{\alpha}}\right)^{2} d F(\lambda)\right)^{m} d F_{l}(u)\right)^{\frac{1}{m}} \\
& \leq \sum_{k=1}^{M} \frac{1}{k^{2 \alpha}} \cdot 2 \cdot 4^{2(1-\alpha)} t^{2 \alpha} \pi^{2 \alpha} \\
& \quad \times \sum_{l=0}^{N-2}\left|\lambda_{l+1}-\lambda_{l}\right|^{2 \alpha}\left(b_{l}^{2}+\left(\frac{t(1+C)}{2}\right)^{2 \alpha} \int_{\lambda_{l}}^{\lambda_{l+1}} \lambda^{2 \alpha} d F(\lambda)\right) \\
& \quad+4 \sum_{k=1}^{M}(F(+\infty)-F(\Lambda)) .
\end{aligned}
$$

The sum $\sum_{k=1}^{M} \frac{1}{k^{2 \alpha}}$ with $\frac{1}{2}<\alpha \leq 1$ can be estimated as follows:

$$
\begin{aligned}
\sum_{k=1}^{M} \frac{1}{k^{2 \alpha}} & \leq 1+\sum_{k=2}^{M} \int_{k-1}^{k} \frac{1}{x^{2 \alpha}} d x=1+\int_{1}^{M} \frac{1}{x^{2 \alpha}} d x=1+\left.\frac{x^{1-2 \alpha}}{1-2 \alpha}\right|_{1} ^{M} \\
& =\frac{2 \alpha}{2 \alpha-1}-\frac{1}{(2 \alpha-1) M^{2 \alpha-1}} .
\end{aligned}
$$

Then

$$
\begin{aligned}
\tau^{2}\left(\sum_{k=1}^{M} \cos (k x) \sum_{l=0}^{N-1} \int_{\lambda_{l}}^{\lambda_{l+1}}\left(J_{k}(t \lambda)-J_{k}\left(t \zeta_{l}\right)\right) d \eta_{1, k}(\lambda)\right) \\
\leq \frac{1}{2 \alpha-1}\left(2 \alpha-\frac{1}{M^{2 \alpha-1}}\right) 2 \cdot 4^{2(1-\alpha)} t^{2 \alpha} \pi^{2 \alpha} \\
\quad \times \sum_{l=0}^{N-2}\left|\lambda_{l+1}-\lambda_{l}\right|^{2 \alpha}\left(b_{l}^{2}+\left(\frac{t(1+C)}{2}\right)^{2 \alpha} \int_{\lambda_{l}}^{\lambda_{l+1}} \lambda^{2 \alpha} d F(\lambda)\right) \\
+4 M(F(+\infty)-F(\Lambda)) .
\end{aligned}
$$

The second inequality is proved similarly. 
Lemma 4.4. Let the integral $\int_{0}^{\infty} \lambda^{2 \alpha} d F(\lambda)<\infty$ converge for $\frac{1}{2}<\alpha \leq 1$. Then

$$
\begin{aligned}
& \tau^{2}\left(\sum_{k=M+1}^{\infty} \cos (k x) \int_{0}^{\infty} J_{k}(t \lambda) d \eta_{1, k}(\lambda)\right) \\
& \leq 2^{2(1-\alpha)} t^{2 \alpha} \pi^{2 \alpha} \frac{1}{(2 \alpha-1) M^{2 \alpha-1}}\left(\int_{0}^{\infty} \lambda^{2 \alpha} d F(\lambda)\right), \\
& \tau^{2}\left(\sum_{k=M+1}^{\infty} \sin (k x) \int_{0}^{\infty} J_{k}(t \lambda) d \eta_{2, k}(\lambda)\right) \\
& \leq 2^{2(1-\alpha)} t^{2 \alpha} \pi^{2 \alpha} \frac{1}{(2 \alpha-1) M^{2 \alpha-1}}\left(\int_{0}^{\infty} \lambda^{2 \alpha} d F(\lambda)\right) .
\end{aligned}
$$

Proof. Indeed,

$$
\begin{aligned}
& \tau^{2}\left(\sum_{k=M+1}^{\infty} \cos (k x) \int_{0}^{\infty} J_{k}(t \lambda) d \eta_{1, k}(\lambda)\right) \\
& \leq \sum_{k=M+1}^{\infty} \tau^{2}\left(\int_{0}^{\infty} J_{k}(t \lambda) d \eta_{1, k}(\lambda)\right) \leq \sum_{k=M+1}^{\infty} \theta^{2}\left(\int_{0}^{\infty} J_{k}(t \lambda) d \eta_{1, k}(\lambda)\right) \\
& =\sum_{k=M+1}^{\infty} \sup _{m \geq 1}\left[\frac{2^{m} \cdot m !}{(2 m) !} \mathrm{E}\left(\int_{0}^{\infty} J_{k}(t \lambda) d \eta_{1, k}(\lambda)\right)^{2 m}\right]^{\frac{1}{m}} .
\end{aligned}
$$

Applying Lemma 4.1,

$$
\begin{aligned}
\mathrm{E}\left(\int_{0}^{\infty} J_{k}(t \lambda) d \eta_{1, k}(\lambda)\right)^{2 m} & \leq \frac{(2 m) !}{2^{m} \cdot m !}\left(\int_{0}^{\infty}\left|J_{k}(t \lambda)\right|^{2} d F(\lambda)\right)^{m} \\
& \leq \frac{(2 m) !}{2^{m} \cdot m !}\left(\int_{0}^{\infty}\left(2^{1-\alpha}|t \lambda|^{\alpha} \pi^{\alpha} \cdot \frac{1}{k^{\alpha}}\right)^{2} d F(\lambda)\right)^{m} \\
& =\frac{(2 m) !}{2^{m} \cdot m !} \cdot \frac{2^{2 m(1-\alpha)} t^{2 m \alpha} \pi^{2 m \alpha}}{k^{2 m \alpha}}\left(\int_{0}^{\infty} \lambda^{2 \alpha} d F(\lambda)\right)^{m}
\end{aligned}
$$

whence

$\tau^{2}\left(\sum_{k=M+1}^{\infty} \cos (k x) \int_{0}^{\infty} J_{k}(t \lambda) d \eta_{1, k}(\lambda)\right) \leq 2^{2(1-\alpha)} t^{2 \alpha} \pi^{2 \alpha} \sum_{k=M+1}^{\infty} \frac{1}{k^{2 \alpha}}\left(\int_{0}^{\infty} \lambda^{2 \alpha} d F(\lambda)\right)$.

The tail $\sum_{k=M+1}^{\infty} \frac{1}{k^{2 \alpha}}$ with $\frac{1}{2}<\alpha \leq 1$ can be estimated as follows:

$$
\sum_{k=M+1}^{\infty} \frac{1}{k^{2 \alpha}} \leq \sum_{k=M+1}^{\infty} \int_{k-1}^{k} \frac{1}{x^{2 \alpha}} d x=\int_{M}^{\infty} \frac{1}{x^{2 \alpha}} d x=\left.\frac{x^{1-2 \alpha}}{1-2 \alpha}\right|_{M} ^{\infty}=\frac{1}{(2 \alpha-1) M^{2 \alpha-1}} .
$$

Thus

$$
\begin{aligned}
& \tau^{2}\left(\sum_{k=M+1}^{\infty} \cos (k x) \int_{0}^{\infty} J_{k}(t \lambda) d \eta_{1, k}(\lambda)\right) \\
& \quad \leq 2^{2(1-\alpha)} t^{2 \alpha} \pi^{2 \alpha} \frac{1}{(2 \alpha-1) M^{2 \alpha-1}}\left(\int_{0}^{\infty} \lambda^{2 \alpha} d F(\lambda)\right) .
\end{aligned}
$$

The second inequality is proved similarly. 
Theorem 4.1. Let $X(t, x)$ and $\hat{X}(t, x)$ be defined by (11) and (2), respectively. Assume that the integral $\int_{0}^{\infty} \lambda^{2 \alpha} d F(\lambda)$ with $\frac{1}{2}<\alpha \leq 1$ converges. Then

$$
\begin{aligned}
\tau^{2}(X(t, x)-\hat{X}(t, x)) \leq & \frac{4}{2 \alpha-1}\left(2 \alpha-\frac{1}{M^{2 \alpha-1}}\right) 2 \cdot 4^{2(1-\alpha)} t^{2 \alpha} \pi^{2 \alpha} \\
& \times \sum_{l=0}^{N-2}\left|\lambda_{l+1}-\lambda_{l}\right|^{2 \alpha}\left(b_{l}^{2}+\left(\frac{t(1+C)}{2}\right)^{2 \alpha} \int_{\lambda_{l}}^{\lambda_{l+1}} \lambda^{2 \alpha} d F(\lambda)\right) \\
& +16 M(F(+\infty)-F(\Lambda)) \\
+ & 2^{2(1-\alpha)} t^{2 \alpha} \pi^{2 \alpha} \frac{4}{(2 \alpha-1) M^{2 \alpha-1}}\left(\int_{0}^{\infty} \lambda^{2 \alpha} d F(\lambda)\right),
\end{aligned}
$$

where $C=\max _{0<l \leq N-2} \frac{\lambda_{l+1}}{\lambda_{l}}$.

Proof. The proof of Theorem 4.1 follows from relations (3) and (4) in view of Lemmas 4.3 and 4.4 .

\section{ACCURACY AND RELIABILITY OF MODELS FOR RANDOM FIELDS}

Definition $5.1([5])$. Let $\{\mathbb{T}, \mathfrak{B}, \mu\}$ be a measurable space and let $X$ and $\hat{X}$ be two random fields defined on $\mathbb{T}$. We say that $\hat{X}(t)$ approximates $X(t)$ with reliability $1-\delta$, $0<\delta<1$, and accuracy $\varepsilon>0$ in the space $L_{p}(\mathbb{T})$ if

$$
\mathrm{P}\left\{\left(\int_{\mathbb{T}}|X(t)-\hat{X}(t)|^{p} d \mu(t)\right)^{\frac{1}{p}}>\varepsilon\right\} \leq \delta .
$$

Theorem 5.1. Let $X=\{X(t), t \in \mathbb{T}\}$ be a sub-Gaussian random field such that

$$
\mathrm{E} X(t)=0, \quad \tau^{2}(t)=\tau^{2}(X(t))=\mathrm{E}(X(t))^{2} .
$$

Assume that

$$
\int_{\mathbb{T}}\left(\mathrm{E}(X(t))^{2}\right)^{\frac{p}{2}} d \mu(t)<\infty, \quad p \geq 1
$$

Then

with probability one. Moreover

$$
\int_{\mathbb{T}}|X(t)|^{p} d \mu(t)<\infty
$$

$$
\mathrm{P}\left\{\|X(t)\|_{L_{p}}>\varepsilon\right\} \leq 2 \exp \left\{-\frac{\varepsilon^{2}}{2 c_{p}^{\frac{2}{p}}}\right\}
$$

for all $\varepsilon$ such that $\varepsilon>c_{p}^{\frac{1}{p}} p^{\frac{1}{2}}$, where $c_{p}=\int_{\mathbb{T}}(\tau(t))^{p} d \mu(t)$.

Theorem 5.1 is a partial case of Theorem 2.1 in $[18$.

Theorem 5.2. Let $\frac{1}{2}<\alpha \leq 1$ and let

$$
\int_{0}^{\infty} \lambda^{2 \alpha} d F(\lambda)<\infty
$$

Assume that a partition $L$ used to construct a model $\hat{X}(t, x), t \in[0, T], x \in[0,2 \pi]$, according to (2) is such that

$$
I \leq \frac{\varepsilon^{p}}{\max \left(\left(2 \ln \frac{2}{\delta}\right)^{\frac{p}{2}}, p^{\frac{p}{2}}\right)}
$$


where

$$
\begin{aligned}
I= & \frac{T^{p \alpha+1}}{p \alpha+1}\left(\frac{2^{p} D_{p}^{3}}{(2 \alpha-1)^{\frac{p}{2}}}\left(2 \alpha-\frac{1}{M^{2 \alpha-1}}\right)^{\frac{p}{2}} 2^{\frac{p}{2}+1} \cdot 4^{p(1-\alpha)} \pi^{p \alpha+1}\left(\sum_{l=0}^{N-2}\left|\lambda_{l+1}-\lambda_{l}\right|^{2 \alpha} b_{l}^{2}\right)^{\frac{p}{2}}\right. \\
& \left.+D_{p} 2^{p(1-\alpha)+1} \pi^{p \alpha+1}\left(\frac{4}{(2 \alpha-1) M^{2 \alpha-1}}\right)^{\frac{p}{2}}\left(\int_{0}^{\infty} \lambda^{2 \alpha} d F(\lambda)\right)^{\frac{p}{2}}\right) \\
& +\frac{T^{2 p \alpha+1}}{2 p \alpha+1} \frac{2^{p} D_{p}^{3}}{(2 \alpha-1)^{\frac{p}{2}}}\left(2 \alpha-\frac{1}{M^{2 \alpha-1}}\right)^{\frac{p}{2}} 2^{\frac{p}{2}+1} \cdot 4^{p(1-\alpha)} \pi^{p \alpha+1}\left(\frac{1+C}{2}\right)^{p \alpha} \\
& \times\left(\sum_{l=0}^{N-2}\left|\lambda_{l+1}-\lambda_{l}\right|^{2 \alpha} \cdot \int_{\lambda_{l}}^{\lambda_{l+1}} \lambda^{2 \alpha} d F(\lambda)\right)^{\frac{p}{2}} \\
+ & T \cdot 2^{2 p+1} \pi D_{p}^{2} M^{\frac{p}{2}}(F(+\infty)-F(\Lambda))^{\frac{p}{2}}
\end{aligned}
$$

and

$$
C=\max _{0<l \leq N-2} \frac{\lambda_{l+1}}{\lambda_{l}}, \quad D_{p}= \begin{cases}1, & \text { if } 0<\frac{p}{2} \leq 1, \\ 2^{\frac{p}{2}-1}, & \text { if } \frac{p}{2}>1 .\end{cases}
$$

Then the model $\hat{X}(t, x)$ approximates the Gaussian field $X(t, x)$ with reliability $1-\delta$, $0<\delta<1$, and accuracy $\varepsilon>0$ in the space $L_{p}(\mathbb{T}), p \geq 1$.

Proof. If

$$
\varepsilon>\left(\int_{0}^{T} \int_{0}^{2 \pi}(\tau(X(t, x)-\hat{X}(t, x)))^{p} d x d t\right)^{\frac{1}{p}} \cdot p^{\frac{1}{2}},
$$

then Theorem 5.1 and Definition 5.1 imply that

$$
\mathrm{P}\left\{\|X(t, x)-\hat{X}(t, x)\|_{L_{p}}>\varepsilon\right\} \leq 2 \exp \left\{-\frac{\varepsilon^{2}}{2 c_{p}^{\frac{2}{p}}}\right\} \leq \delta
$$

where

$$
c_{p}=\int_{0}^{T} \int_{0}^{2 \pi}(\tau(X(t, x)-\hat{X}(t, x)))^{p} d x d t .
$$

The latter inequality holds if

$$
\int_{0}^{T} \int_{0}^{2 \pi}(\tau(X(t, x)-\hat{X}(t, x)))^{p} d x d t \leq \frac{\varepsilon^{p}}{\left(2 \ln \frac{2}{\delta}\right)^{\frac{p}{2}}} .
$$

Since

$$
(a+b)^{\frac{p}{2}} \leq D_{p}\left(a^{\frac{p}{2}}+b^{\frac{p}{2}}\right)
$$

with the constant

$$
D_{p}= \begin{cases}1, & \text { if } \frac{p}{2} \leq 1 \\ 2^{\frac{p}{2}-1}, & \text { if } \frac{p}{2}>1\end{cases}
$$


Theorem 4.1 implies that

$$
\begin{aligned}
& (\tau(X(t, x)-\hat{X}(t, x)))^{p} \\
& \leq\left[\frac{4}{2 \alpha-1}\left(2 \alpha-\frac{1}{M^{2 \alpha-1}}\right) 2 \cdot 4^{2(1-\alpha)} t^{2 \alpha} \pi^{2 \alpha}\right. \\
& \times \sum_{l=0}^{N-2}\left|\lambda_{l+1}-\lambda_{l}\right|^{2 \alpha}\left(b_{l}^{2}+\left(\frac{t(1+C)}{2}\right)^{2 \alpha} \int_{\lambda_{l}}^{\lambda_{l+1}} \lambda^{2 \alpha} d F(\lambda)\right) \\
& +16 M(F(+\infty)-F(\Lambda)) \\
& \left.+2^{2(1-\alpha)} t^{2 \alpha} \pi^{2 \alpha} \frac{4}{(2 \alpha-1) M^{2 \alpha-1}}\left(\int_{0}^{\infty} \lambda^{2 \alpha} d F(\lambda)\right)\right]^{\frac{p}{2}} \\
& \leq D_{p}\left(\frac{4}{2 \alpha-1}\left(2 \alpha-\frac{1}{M^{2 \alpha-1}}\right) 2 \cdot 4^{2(1-\alpha)} t^{2 \alpha} \pi^{2 \alpha}\right. \\
& \times \sum_{l=0}^{N-2}\left|\lambda_{l+1}-\lambda_{l}\right|^{2 \alpha}\left(b_{l}^{2}+\left(\frac{t(1+C)}{2}\right)^{2 \alpha} \int_{\lambda_{l}}^{\lambda_{l+1}} \lambda^{2 \alpha} d F(\lambda)\right) \\
& +16 M(F(+\infty)-F(\Lambda)))^{\frac{p}{2}} \\
& +D_{p} 2^{p(1-\alpha)} t^{p \alpha} \pi^{p \alpha}\left(\frac{4}{(2 \alpha-1) M^{2 \alpha-1}}\right)^{\frac{p}{2}}\left(\int_{0}^{\infty} \lambda^{2 \alpha} d F(\lambda)\right)^{\frac{p}{2}} \\
& \leq D_{p}^{2}\left(\left(\frac{4}{2 \alpha-1}\right)^{\frac{p}{2}}\left(2 \alpha-\frac{1}{M^{2 \alpha-1}}\right)^{\frac{p}{2}} 2^{\frac{p}{2}} \cdot 4^{p(1-\alpha)} t^{p \alpha} \pi^{p \alpha}\right. \\
& \times\left(\sum_{l=0}^{N-2}\left|\lambda_{l+1}-\lambda_{l}\right|^{2 \alpha}\left(b_{l}^{2}+\left(\frac{t(1+C)}{2}\right)^{2 \alpha} \int_{\lambda_{l}}^{\lambda_{l+1}} \lambda^{2 \alpha} d F(\lambda)\right)\right)^{\frac{p}{2}} \\
& \left.+4^{p} M^{\frac{p}{2}}(F(+\infty)-F(\Lambda))^{\frac{p}{2}}\right) \\
& +D_{p} 2^{p(1-\alpha)} t^{p \alpha} \pi^{p \alpha}\left(\frac{4}{(2 \alpha-1) M^{2 \alpha-1}}\right)^{\frac{p}{2}}\left(\int_{0}^{\infty} \lambda^{2 \alpha} d F(\lambda)\right)^{\frac{p}{2}} \\
& \leq D_{p}^{2}\left(\frac{2^{p} D_{p}}{(2 \alpha-1)^{\frac{p}{2}}}\left(2 \alpha-\frac{1}{M^{2 \alpha-1}}\right)^{\frac{p}{2}} 2^{\frac{p}{2}} \cdot 4^{p(1-\alpha)} t^{p \alpha} \pi^{p \alpha}\right. \\
& \times\left(\left(\sum_{l=0}^{N-2}\left|\lambda_{l+1}-\lambda_{l}\right|^{2 \alpha} b_{l}^{2}\right)^{\frac{p}{2}}\right. \\
& \left.+t^{p \alpha}\left(\frac{1+C}{2}\right)^{p \alpha}\left(\sum_{l=0}^{N-2}\left|\lambda_{l+1}-\lambda_{l}\right|^{2 \alpha} \int_{\lambda_{l}}^{\lambda_{l+1}} \lambda^{2 \alpha} d F(\lambda)\right)^{\frac{p}{2}}\right) \\
& \left.+4^{p} M^{\frac{p}{2}}(F(+\infty)-F(\Lambda))^{\frac{p}{2}}\right)
\end{aligned}
$$




$$
+D_{p} 2^{p(1-\alpha)} t^{p \alpha} \pi^{p \alpha}\left(\frac{4}{(2 \alpha-1) M^{2 \alpha-1}}\right)^{\frac{p}{2}}\left(\int_{0}^{\infty} \lambda^{2 \alpha} d F(\lambda)\right)^{\frac{p}{2}} .
$$

Therefore

$$
\begin{aligned}
& (\tau(X(t, x)-\hat{X}(t, x)))^{p} \\
& \leq\left(\frac{2^{p} D_{p}^{3}}{(2 \alpha-1)^{\frac{p}{2}}}\left(2 \alpha-\frac{1}{M^{2 \alpha-1}}\right)^{\frac{p}{2}} 2^{\frac{p}{2}} \cdot 4^{p(1-\alpha)} \pi^{p \alpha}\left(\sum_{l=0}^{N-2}\left|\lambda_{l+1}-\lambda_{l}\right|^{2 \alpha} b_{l}^{2}\right)^{\frac{p}{2}}\right. \\
& \left.\quad+D_{p} 2^{p(1-\alpha)} \pi^{p \alpha}\left(\frac{4}{(2 \alpha-1) M^{2 \alpha-1}}\right)^{\frac{p}{2}}\left(\int_{0}^{\infty} \lambda^{2 \alpha} d F(\lambda)\right)^{\frac{p}{2}}\right) t^{p \alpha} \\
& \left.\left.+\frac{2^{p} D_{p}^{3}}{(2 \alpha-1)^{\frac{p}{2}}}\left(2 \alpha-\frac{1}{M^{2 \alpha-1}}\right)^{\frac{p}{2}} 2^{\frac{p}{2}} \cdot 4^{p(1-\alpha)} \pi^{p \alpha}\left(\frac{1+C}{2}\right)^{p \alpha}\right)^{N-2} \mid \sum_{l=0}^{\lambda_{l+1}} \lambda^{2 \alpha} d F(\lambda)\right)^{\frac{p}{2}} t^{2 p \alpha} \\
& +4^{p} D_{p}^{2} M^{\frac{p}{2}}(F(+\infty)-F(\Lambda))^{\frac{p}{2}} .
\end{aligned}
$$

Then

$$
\begin{aligned}
& \int_{0}^{T} \int_{0}^{2 \pi}(\tau(X(t, x)-\hat{X}(t, x)))^{p} d x d t \frac{T^{p \alpha+1}}{p \alpha+1}\left(\frac{2^{p} D_{p}^{3}}{(2 \alpha-1)^{\frac{p}{2}}}\left(2 \alpha-\frac{1}{M^{2 \alpha-1}}\right)^{\frac{p}{2}}\right. \\
& \quad \times 2^{\frac{p}{2}+1} \cdot 4^{p(1-\alpha)} \pi^{p \alpha+1}\left(\sum_{l=0}^{N-2}\left|\lambda_{l+1}-\lambda_{l}\right|^{2 \alpha} b_{l}^{2}\right)^{\frac{p}{2}} \\
&\left.\quad+D_{p} 2^{p(1-\alpha)+1} \pi^{p \alpha+1}\left(\frac{4}{(2 \alpha-1) M^{2 \alpha-1}}\right)^{\frac{p}{2}}\left(\int_{0}^{\infty} \lambda^{2 \alpha} d F(\lambda)\right)^{\frac{p}{2}}\right) \\
&+\frac{T^{2 p \alpha+1}}{2 p \alpha+1} \frac{2^{p} D_{p}^{3}}{(2 \alpha-1)^{\frac{p}{2}}}\left(2 \alpha-\frac{1}{M^{2 \alpha-1}}\right)^{\frac{p}{2}} 2^{\frac{p}{2}+1} \cdot 4^{p(1-\alpha)} \pi^{p \alpha+1}\left(\frac{1+C}{2}\right)^{p \alpha} \\
& \quad \times\left(\sum_{l=0}^{N-2}\left|\lambda_{l+1}-\lambda_{l}\right|^{2 \alpha} \cdot \int_{\lambda_{l}}^{\lambda_{l+1}} \lambda^{2 \alpha} d F(\lambda)\right)^{\frac{p}{2}} \\
&+T \cdot 2^{2 p+1} \pi D_{p}^{2} M^{\frac{p}{2}}(F(+\infty)-F(\Lambda))^{\frac{p}{2}} \\
&=I . \quad
\end{aligned}
$$

Corollary 5.1. Let a partition $L=\left\{\lambda_{0}, \ldots, \lambda_{N}\right\}$ of the set $[0, \infty)$ be such that $\lambda_{l}<\lambda_{l+1}$ and $\lambda_{l+1}-\lambda_{l}=\frac{\Lambda}{N-1}$. Then Theorem 5.2 holds with

$$
I=\left(\frac{\Lambda}{N-1}\right)^{p \alpha} \cdot A+\left(\frac{1}{M^{2 \alpha-1}}\right)^{\frac{p}{2}} \cdot B+(F(+\infty)-F(\Lambda))^{\frac{p}{2}} \cdot H
$$


where

$$
\begin{gathered}
A=2^{p} D_{p}^{3}\left(\frac{2 \alpha}{2 \alpha-1}\right)^{\frac{p}{2}} 2^{\frac{p}{2}+1} \cdot 4^{p(1-\alpha)} \pi^{p \alpha+1} \\
\times\left(\frac{T^{p \alpha+1}}{p \alpha+1}+\left(\frac{3}{2}\right)^{p \alpha}\left(\int_{0}^{\Lambda} \lambda^{2 \alpha} d F(\lambda)\right)^{\frac{p}{2}} \frac{T^{2 p \alpha+1}}{2 p \alpha+1}\right), \\
B=\frac{2^{p} D_{p}}{(2 \alpha-1)^{\frac{p}{2}}} 2^{p(1-\alpha)+1} \pi^{p \alpha+1}\left(\int_{0}^{\infty} \lambda^{2 \alpha} d F(\lambda)\right)^{\frac{p}{2}} \cdot \frac{T^{p \alpha+1}}{p \alpha+1} \\
H=2^{2 p+1} \cdot D_{p}^{2} \cdot \pi \cdot M^{\frac{p}{2}} \cdot T .
\end{gathered}
$$

\section{Concluding Remarks}

A model for a homogeneous isotropic Gaussian random field is constructed. Bounds are found for the probability that a model approximates a random field with a given accuracy and reliability in the space $L_{p}(\mathbb{T}), p \geq 1$.

\section{BIBLIOGRAPHY}

1. S. M. Ermakov and G. A. Mikhaı̌lov, A Course in Statistical Modeling, "Nauka", Moscow, 1982. (Russian) MR705787 (85h:65014)

2. V. A. Ogorodnikov and S. M. Prigarin, Numerical Modeling of Random Processes and Fields: Algorithms and Applications, VSP, Utrecht, 1996. MR1419502 (97k:65012)

3. Yu. Kozachenko, T. Sottinen, and O. Vasylyk, Simulation of weakly self-similar stationary increment $S_{u} b_{\varphi}(\Omega)$-processes: a series expansion approach, Methodology and Computing in Applied Probability 7 (2005), no. 3, 379-400. MR2210587(2008i:60072)

4. K. K. Sabelfeld and O. A. Kurbanmuradov, Numerical statistical model of classical incompressible isotropic turbulence, Soviet Journal of Numerical Analysis and Mathematical Modeling 5 (1990), 251-263. MR1122132 (92k:76043)

5. Yu. V. Kozachenko, A. O. Pashko, and I. V. Rozora, Modeling of Random Processes and Fields, "Zadruga", Kyiv, 2007. (Ukrainian)

6. A. M. Tegza and N. V. Fedoryanych, Accuracy and reliability of a model of a Gaussian homogeneous and isotropic random field in the space $C(T)$ with a bounded spectrum, Naukovyi Visnyk Uzhgorod University 22 (2011), no. 2, 142-147. (Ukrainian)

7. Yu. Kozachenko and I. Rozora, Simulation of Gaussian stochastic processes, Random Oper. Stoch. Equ. 11 (2003), no. 3, 275-296. MR2009187 (2004i:60050)

8. Yu. V. Kozachenko, I. V. Rozora, and Ye. V. Turchyn, On an expansion of random processes in series, Random Oper. Stoch. Equ. 15 (2007), no. 1, 15-33. MR2316186 (2008a:60131)

9. Yu. V. Kozachenko and A. M. Tegza, Application of the theory of $\operatorname{Sub}_{\varphi}(\Omega)$ spaces of random variables for determining the accuracy of the modeling of stationary Gaussian processes, Teor. Imovir. Mat. Stat. 67 (2002), 71-87; English transl. in Theory Probab. Math. Statist. 67 (2003), 79-96. MR 1956621(2003j:60049)

10. N. V. Troshki, Construction of models of Gaussian random fields with a given reliability and accuracy in $L_{p}(\mathbb{T}), p \geq 1$, Appl. Stat. Actuarial and Finance Math. (2013), no. 1-2, 149-156. (Ukrainian)

11. Yu. V. Kozachenko and O. O. Pogoriliak, Simulation of Cox processes driven by random Gaussian field, Methodology and Computing Appl. Probab. 13 (2011), no. 3, 511-521. MR2822393

12. Yu. V. Kozachenko and O. M. Moklyachuk, Stochastic processes in the spaces $D_{V, W}$, Teor. Imovir. Mat. Stat. 82 (2010), 56-66; English transl. in Theory Probab. Math. Statist. 82 (2011), 43-56. MR2790483(2011m:60101)

13. Yu. V. Kozachenko and O. M. Moklyachuk, Sample continuity and modeling of stochastic processes from the spaces $D_{V, W}$, Teor. Imovir. Mat. Stat. 83 (2010), 80-91; English transl. in Theory Probab. Math. Statist. 83 (2011), 95-110. MR2768851 (2011k:60115)

14. Yu. V. Kozachenko and Yu. Yu. Mlavets', The Banach spaces $F_{\psi}(\Omega)$ of random variables, Teor. Imovir. Mat. Stat. 86 (2012), 92-107; English transl. in Theory Probab. Math. Statist. 86 (2013), 105-121. MR2986453 
15. V. V. Buldygin and Yu. V. Kozachenko, Metric Characterization of Random Variables and Random Processes, "TViMS", Kyiv, 1998; English transl., American Mathematical Society, Providence, RI, 2000. MR.1743716 (2001g:60089)

16. M. I. Yadrenko, Spectral Theory of Random Fields, "Vyshcha shkola", Kiev, 1980; English transl., Optimization Software, Inc., Publications Division, New York-Heidelberg-Berlin, 1983. MR.590889 (82e:60001)

17. H. Bateman and A. Erdelyi, Higher Transcendental Functions, vol. 2, McGraw-Hill, New YorkToronto-London, 1953. MR0058756(15:419i)

18. Yu. V. Kozachenko and O. E. Kamenshchikova, Approximation of $\operatorname{SSub}_{\varphi}(\Omega)$ stochastic processes in the space $L_{p}(\mathbb{T})$, Teor. Imovir. Mat. Stat. 79 (2008), 73-78; English transl. in Theory Probab. Math. Statist. 79 (2009), 83-88. MR2494537(2010d:60097)

Department of Probability Theory, Statistics, and Actuarial Mathematics, Faculty for Mechanics and Mathematics, National Taras Shevchenko University, Volodymyrs'ka Street, 64, KYIV 01601, UKRAINE

E-mail address: FedoryanichNatali@ukr.net

Received 11/MAR/2014

Translated by N. SEMENOV 